\title{
Therapeutic properties of the new phytochemical osmotin for preventing atherosclerosis
}

\author{
Takuya Watanabe \\ Department of Internal Medicine, Ushioda General Hospital/Clinic, Yokohama 230-0001, Japan.
}

Correspondence to: Dr. Takuya Watanabe, Department of Internal Medicine, Ushioda General Hospital/Clinic, Yokohama Workers' Welfare Association, 1-6-20 Yako, Tsurumi-ku, Yokohama 230-0001, Japan. E-mail: t-watanabe@ushioda.or.jp

How to cite this article: Watanabe T. Therapeutic properties of the new phytochemical osmotin for preventing atherosclerosis. Vessel Plus 2020;4:4. http://dx.doi.org/10.20517/2574-1209.2019.27

Received: 30 Aug 2019 First Decision: 9 Jan 2020 Revised: 5 Feb 2020 Accepted: 2 Mar 2020 Published: 13 Mar 2020

Science Editor: Ali H. Eid Copy Editor: Jing-Wen Zhang Production Editor: Jing Yu

\begin{abstract}
Osmotin, a natural plant protein found in tomato, potato, pepper, and tobacco, is a homolog of human adiponectin. It exerts multiple biological activities through adiponectin receptors in a variety of mammalian cells. The therapeutic properties of osmotin have recently been shown in the pathogenesis of atherosclerosis by in vitro and in vivo experiments. Osmotin suppresses the adhesion of monocytes to endothelial cells by downregulating inflammatory cytokines and adhesion molecules in both cells. It suppresses oxidized low-density lipoprotein-induced foam cell formation by downregulating cluster of differentiation 36 and acyl-coenzyme A:cholesterol acyltransferase 1 as well as upregulating ATP-binding cassette transporter A1 in monocyte-derived macrophages. In vascular smooth muscle cells, osmotin suppresses the migration, proliferation, and production of collagen 1, fibronectin, and matrix metalloproteinase 2 by decreasing the phosphorylation of extracellular signal-regulated protein kinase $1 / 2$ and nuclear factor- $\mathrm{KB}$ as well as increasing AMP-activated protein kinase (AMPK) expression. Treatment with osmotin suppresses abdominal fat accumulation in C57BL/6 mice and prevents the development of aortic atherosclerotic lesions, improving vascular inflammation and plaque instability in apolipoprotein E-deficient $($ Apoe-/) mice. Osmotin protects against obesity- and diabetes-induced nonalcoholic fatty liver disease in leptin-deficient obese $(o b / o b)$ and leptin receptor-deficient diabetic $(d b / d b)$ mice. These effects are attributed to the stimulatory actions of osmotin on peroxisome proliferator-activated receptor- $\alpha$ and AMPK. Moreover, osmotin lowers serum levels of total cholesterol and triglyceride in non-diabetic and diabetic rats. These findings suggest that osmotin contributes to improving the extracellular risk factors for atherosclerosis and vascular intracellular and molecular responses. Therefore, the novel phytochemical osmotin may serve as a novel therapeutic target for atherosclerosis and related diseases.
\end{abstract}


Keywords: Osmotin, atherosclerosis, inflammation, diabetes, obesity, phytochemical, vascular cells, animal models

\section{INTRODUCTION}

Atherosclerosis is clinically manifested as coronary artery disease, stroke, and peripheral arterial disease, which are major causes of mortality and morbidity worldwide ${ }^{[1]}$. Atherosclerosis is a chronic inflammatory vascular disease induced by endothelial injury followed by atheromatous plaque formation, leading to thickening and loss of elasticity in the arterial wall of medium- and large-sized arteries, including the coronary, cerebral, and carotid arteries and the aorta ${ }^{[2]}$. Risk factors of atherosclerosis include dyslipidemia, diabetes, obesity, and hypertension. The pathophysiology of atheromatous plaque development involves endothelial cell (EC) inflammation and proliferation, monocyte adhesion to ECs and infiltration into the under-endothelial space, inflammatory cytokine release from monocyte-derived macrophages, oxidized low-density lipoprotein (Ox-LDL)-induced macrophage foam cell formation, vascular smooth muscle cell (VSMC) migration and proliferation, and extracellular matrix (ECM) production by VSMCs ${ }^{[3]}$. The progression and rupture of atheromatous plaques in coronary arteries lead to myocardial ischemia and infarction. Timely reperfusion is critical for the salvage of ischemic myocardium. After coronary angioplasty, restoration of blood flow to the damaged myocardium triggers further ischemic myocardial damage. This paradoxical phenomenon is known as ischemia-reperfusion injury, which is a serious clinical problem in the treatment of patients with coronary artery disease. Several studies have investigated preventive effects of plants on atherogenesis ${ }^{[4-9]}$. Osmotin, a plant-derived natural protein, is receiving the most attention as a therapeutic target for atherosclerosis and myocardial ischemia-reperfusion injury ${ }^{[1,11]}$.

Osmotin was first isolated from tobacco (Nicotiana tabacum) cells by Singh et al ${ }^{[12]}$. Later, osmotin was also found in other plant species, including tomato, potato, oat, pepper, and grape $e^{[13]}$. Osmotin is a plant peptide hormone, also called phytochemical, which belongs to the fifth class of the group of pathogenesisrelated proteins ${ }^{[14]}$. The osmotin gene $A P 24$ is known to be activated by environmental and phytohormone signals ${ }^{[15]}$. Osmotin plays an important role in the protection against osmotic and oxidative stresses caused by higher salt concentration, cold, and drought ${ }^{[16]}$, and has anti-fungal activity in plants ${ }^{[17]}$. In plants and yeasts, osmotin exhibits anti-fungal, anti-oxidant, and anti-apoptotic effects through $\mathrm{PHO} 36$, which is an adiponectin receptor (AdipoR) homolog ${ }^{[13,18]}$.

Osmotin has recently attracted attention as a homolog of mammalian adiponectin ${ }^{[13,18]}$, which is the most famous adipocytokine (adipokine) with anti-inflammatory, anti-diabetic, and anti-atherogenic properties ${ }^{[19-21]}$. Osmotin is composed of 246 amino acids including a 21 -amino acid signal peptide, which do not share remarkable similarity to human adiponectin (AdipoQ, 244 amino acids) in the amino acid sequence alignment ${ }^{[13]}$. However, the domain I of osmotin is demonstrated to overlap with the $\beta$-barrel domain of AdipoQ by three-dimensional structure analyses ${ }^{[13,18]}$; thereby, osmotin binds to AdipoR1 and then leads to intracellular signaling ${ }^{[18,22,23]}$. Therefore, osmotin is regarded as a natural agonist for human AdipoR $1^{[22]}$. Osmotin exhibits anti-inflammatory and anti-apoptotic effects in mammalian cells through AdipoR $1^{[24,25]}$. Takahashi et al. ${ }^{[10]}$ recently showed that osmotin exerts atheroprotective effects through human AdipoR1 in human vascular cells. In intracellular signaling pathways, both nuclear factor- $\kappa \mathrm{B}(\mathrm{NF}-\kappa \mathrm{B})$ and extracellular signal-regulated protein kinase 1/2 (ERK1/2) suppression and AMP-activated protein kinase (AMPK) activation play a pivotal role in the preventive effects of osmotin on inflammation and atherosclerosis ${ }^{[10]}$.

Osmotin as well as adiponectin binds to AdipoR1, leading to the activation of adaptor protein, phosphotyrosine interacting with PH domain and leucine zipper 1 (APPL1) followed by phosphoinositide 3-kinase (PI3K)/Akt, AMPK, peroxisome proliferator-activated receptor- $\alpha$ (PPAR- $\alpha$ ), and protein-tyrosine 
phosphatase 1B (PTP1B) ${ }^{[10]}$. The PTP1B activation suppresses RAF1 and ERK1/2 phosphorylation ${ }^{[10]}$. The activation of PI3K/Akt and/or AMPK suppresses apoptosis of vascular cells (ECs, VSMCs, and macrophages) and cardiomyocytes. NF- $\mathrm{B}$ and ERK1/2 suppression and AMPK activation inhibit inflammation, differentiation, migration, and proliferation of the vascular cells; thus, these signalings are therapeutic targets for atherosclerosis ${ }^{[26-28]}$. In adipocytes, hepatocytes, and skeletal muscle cells, AMPK and PPAR- $\alpha$ activation contributes to improving insulin resistance and lipid metabolism, respectively. AdipoR1 is expressed at high levels in monocytes, macrophages, ECs, VSMCs, cardiomyocytes, adipocytes, hepatocytes, and skeletal muscle cells ${ }^{[29]}$. The AdipoR agonist AdipoRon, an adiponectin-like synthetic small molecule, is in the spotlight as an oral anti-atherosclerotic drug ${ }^{[30]}$.

Several lines of evidence have recently shown the protective effects of osmotin against atherosclerosis, hyperlipidemia, diabetes, and obesity ${ }^{[10,31-33]}$. This review article summarizes the therapeutic properties of osmotin for preventing atherosclerosis and myocardial ischemia-reperfusion injury as well as inflammation and neurodegeneration. In addition, the article describes the comparisons of the atheroprotective effects between osmotin and adiponectin or AdipoRon. However, there are no data comparing the exact difference in the potency of these effects among the three agents.

\section{ATHEROPROTECTIVE EFFECT}

\section{In vitro anti-atherosclerotic effects of osmotin}

Osmotin has been shown to exert the multiple effects in different cell types including ECs, monocytes/ macrophages, VSMCs, adipocytes, and cardiomyocytes ${ }^{[10,11,33]}$. The anti-atherosclerotic effects of osmotin have been investigated using cultured human vascular cells in vitro. In this section, the evidence regarding the anti-atherosclerotic effects of osmotin is introduced, as summarized in Figure 1.

\section{Preventive effects of osmotin on vascular inflammation}

Osmotin suppresses lipopolysaccharide (LPS)-induced upregulation of monocyte chemoattractant protein 1 (MCP1), tumor necrosis factor- $\alpha$ (TNF- $\alpha$ ), intercellular adhesion molecule 1 (ICAM1), vascular cell adhesion molecule 1 (VCAM1), and E-selectin in human umbilical vein endothelial cells (HUVECs) ${ }^{[10]}$. It suppresses the TNF- $\alpha$-induced adhesion of human THP1 monocytes to HUVECs ${ }^{[10]}$. Osmotin shifts toward an anti-inflammatory phenotype (M2) rather than pro-inflammatory phenotype (M1), associated with ERK1/2 and NF- $\kappa$ B downregulation and PPAR- $\gamma$ upregulation in human THP1 monocyte-derived macrophages ${ }^{[10]}$. It also suppresses the LPS-induced secretion of interleukin 6 (IL6), pentraxin 3 (PTX3), and TNF- $\alpha$ from human THP1 monocyte-derived macrophages ${ }^{[10]}$. These findings indicate that osmotin suppresses vascular inflammation and endothelial dysfunction. In addition, it suppresses the proliferation of human EA.hy926 ECs ${ }^{[10]}$, thus preventing intimal medial thickness. Osmotin mimics the suppressive effects of adiponectin on the expression of ICAM1, VCAM1, and E-selectin in ECs, monocyte-EC adhesion, and EC proliferation as well as inflammatory phenotype (M1) and TNF- $\alpha$ expression in macrophages ${ }^{[34-38]}$.

\section{Preventive effects of osmotin on macrophage foam cell formation}

Osmotin suppresses Ox-LDL-induced accumulation of cholesterol ester (foam cell formation) by downregulating cluster of differentiation 36 (CD36) and acyl-coenzyme A:cholesterol acyltransferase 1 (ACAT1) as well as upregulating ATP-binding cassette transporter A1 (ABCA1) in human THP1 monocyte-derived macrophages ${ }^{[10]}$. These effects are consistent with the effects of adiponectin in suppressing Ox-LDL-induced foam cell formation and ACAT1 expression and enhancing ABCA1 expression in human monocyte-derived macrophages ${ }^{[39-41]}$.

\section{Preventive effects of osmotin on the migration and proliferation of VSMCs}

Osmotin suppresses angiotensin II-induced migration of human aortic smooth muscle cells (HASMCs) ${ }^{[10]}$. It also suppresses the proliferation of HASMCs by decreasing the phosphorylation of RAF1, ERK1/2, 


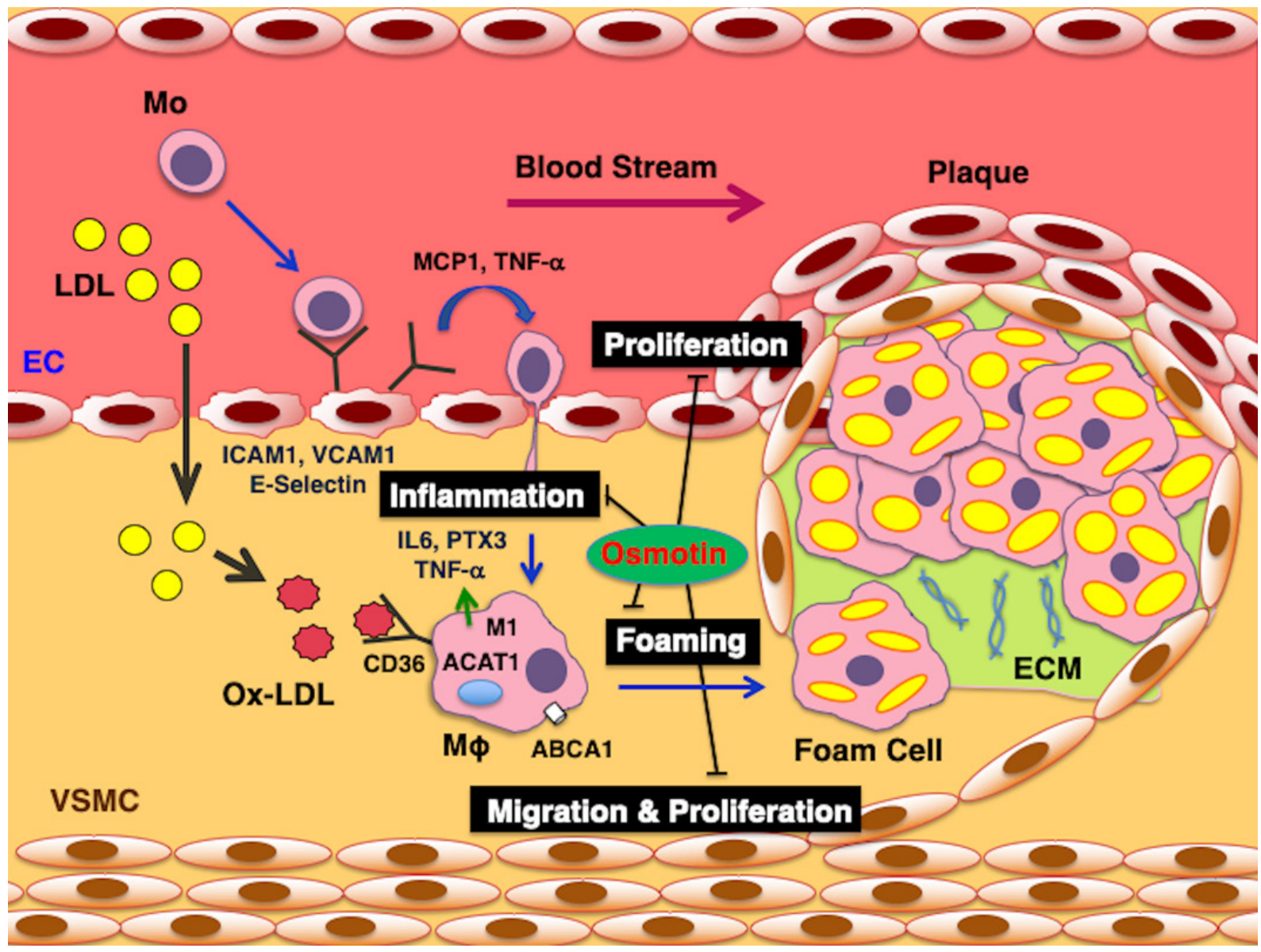

Figure 1. Cellular and molecular mechanisms mediating the preventive effects of osmotin on atherosclerosis. Osmotin suppresses the proliferation of vascular ECs. It suppresses vascular inflammation, characterized as monocyte-EC adhesion, by downregulating MCP1, TNF- $\alpha$, ICAM1, VCAM1, and E-selectin in ECs, and suppresses inflammatory phenotype (M1) and secretion of IL6, PTX3, and TNF- $\alpha$ in monocyte (Mo)-derived macrophages $(\mathrm{M} \phi)$. Osmotin suppresses Ox-LDL-induced foam cell formation by downregulating CD36 and ACAT1 as well as upregulating ABCA1 in Mo-derived macrophages. In VSMCs, osmotin suppresses the migration, proliferation, and production of ECM proteins, such as collagen 1, fibronectin, and matrix metalloproteinase 2 . Therefore, osmotin prevents the development and instability of atheromatous plaques. EC: endothelial cells; MCP1: monocyte chemoattractant protein 1; TNF- $\alpha$ : tumor necrosis factor- $\alpha$; ICAM1: intercellular adhesion molecule 1; VCAM1: vascular cell adhesion molecule 1; IL6: interleukin 6; PTX3: pentraxin 3; OxLDL: oxidized low-density lipoprotein; CD36: cluster of differentiation 36; ACAT1: acyl-coenzyme A:cholesterol acyltransferase 1; ABCA1: ATP-binding cassette transporter A1; VSMCs: vascular smooth muscle cells; ECM: extracellular matrix

and NF- $\mathrm{KB}$ as well as increasing AMPK expression ${ }^{[10]}$. Osmotin exerts the same suppressive effects of adiponectin and AdipoRon on the migration and proliferation of VSMCs ${ }^{[42-44]}$.

Modulatory effects of osmotin on ECM production in VSMCs

The intercellular networking that occurs among ECs, VSMCs, and macrophages leads to a fibroproliferative response, in which ECM plays an important role in atheromatous plaques. The ECM is composed of a mixture of vastly different macromolecules including collagens, fibronectin, elastin, and matrix metalloproteinases (MMPs), which are produced by VSMCs in the arterial wall. Osmotin suppresses the production of collagen 1, fibronectin, and MMP2, and increases that of elastin and MMP9 in the HASMCs $^{[10]}$. The former contributes to preventing the development of atheromatous plaques, while the latter contributes to vascular elasticity and remodeling. However, osmotin has no effect on collagen 3 production in HASMCs ${ }^{[10]}$. Osmotin mimics the suppressive effects of adiponectin on collagen 1 expression in VSMCs ${ }^{[45]}$. 
Suppressive effects of osmotin on the glucose uptake and differentiation of adipocytes

Visceral adipose tissue promotes insulin resistance and metabolic disorders, resulting in the development of atherosclerosis. In particular, perivascular adipose tissue has been recently shown to have a close linkage with atherosclerosis ${ }^{[46]}$. Perivascular adipocytes residing in the vascular adventitia are recognized as endocrine cells ${ }^{[46]}$. Cross-talk between perivascular adipocytes and vascular cells in blood vessel wall modulates the formation of atherosclerosis by releasing adipocytokines ${ }^{[47]}$. Similar to adiponectin ${ }^{[48]}$, osmotin suppresses the differentiation and proliferation of adipocytes by regulating the expression of p21, p27, and cyclin-dependent kinase 2, as well as improves glucose uptake in 3T3-L1 adipocytes ${ }^{[33]}$.

Protective effects of osmotin against ischemia-reperfusion injury in cardiomyocytes

A recent study has shown the protective effects of osmotin against myocardial ischemia-reperfusion injury $^{[11]}$. Osmotin protects rat cardiac myoblast H9c2 cells against ischemia-reperfusion injury through AdipoR1 by increasing phosphorylation of PI3K/Akt and decreasing that of NF- $\kappa \mathrm{B}^{[11]}$. Osmotin exhibits the same cardioprotective effects of adiponectin and AdipoRon against ischemia-reperfusion injury ${ }^{[4,50]}$. These findings indicate that osmotin as well as adiponectin and AdipoRon could prevent myocardial damage following coronary events and ischemia-reperfusion injury.

\section{In vivo anti-atherosclerotic effects of osmotin}

Several studies have shown that adiponectin and AdipoRon suppress the development of atherosclerotic lesions in apolipoprotein E-deficient $\left(A p o e^{-l}\right)$ mice, an atherogenic mouse model, on a normal or high-fat $\operatorname{diet}^{[51-53]}$. Recently, the anti-atherosclerotic effects of osmotin have also been investigated using a variety of animal models in vivo. Treatment with osmotin suppresses abdominal fat accumulation in C57BL/6 mice fed with a high-fat $\operatorname{diet}^{[33]}$. In Apoe ${ }^{-/-}$mice on a high-cholesterol diet, chronic infusion of osmotin prevents the development of aortic atherosclerotic lesions accompanied by an improved vascular inflammation and plaque instability ${ }^{[10]}$. In this model, osmotin also improves fasting plasma glucose level, free fatty acid level, and insulin resistance ${ }^{[10]}$. Similarly, injection of osmotin lowers serum levels of total cholesterol and triglyceride and prevents atherosclerosis in Wistar rats fed with a high-cholesterol diet ${ }^{[31]}$. Osmotin injection decreases serum levels of glucose, insulin, total cholesterol, and triglyceride in streptozotocininduced diabetic rats fed with a high-fat $\operatorname{diet}^{[32]}$. In addition, it protects against obesity and diabetes-induced nonalcoholic fatty liver disease in leptin-deficient obese $(o b / o b)$ mice and leptin receptor-deficient diabetic $(d b / d b)$ mice $^{[54]}$. These effects are attributed to the stimulatory actions of osmotin on AMPK and PPAR- $\alpha$ pathways. Therefore, osmotin is also expected to be useful in the preventive health care in diabetics in future ${ }^{[55]}$.

\section{ANTI-INFLAMMATORY EFFECT}

Atherosclerosis is an inflammatory vascular disease. The Canakinumab Anti-inflammatory Thrombosis Outcome Study trial provided direct evidence that inflammation accelerates cardiovascular disease in humans, by showing that a therapeutic antibody targeting IL1 $\beta$ decreased recurrent cardiovascular events ${ }^{[56]}$. This section introduces the beneficial effects of osmotin on inflammatory diseases other than atherosclerosis. Osmotin suppresses LPS-induced neuroinflammation through AdipoR1 followed by tolllike receptor 4 and $\mathrm{NF}-\kappa \mathrm{B}$ pathways in $\mathrm{BV} 2$ microglial cell ${ }^{[57]}$. In addition, infusion of osmotin using osmotic pumps attenuates dextran sodium sulfate-induced colitis in mice ${ }^{[58]}$. These effects are consistent with anti-inflammatory effects of adiponectin and AdipoRon ${ }^{[58-60]}$. The results from in vitro and in vivo experiments indicate that osmotin as well as adiponectin and AdipoRon could prevent inflammatory diseases.

\section{NEUROPROTECTIVE EFFECT}

Alzheimer's disease is the most common form of dementia. The pathogenesis of Alzheimer's disease involves characteristics such as amyloid- $\beta$ deposition, tau phosphorylation, and apoptotic neurode- 
generation $^{[61]}$. The risk factors of Alzheimer's disease are known to be inflammation, lipid metabolism (Apoe), and atherosclerosis ${ }^{[61-63]}$. A clinical prospective study has shown that atherosclerosis in carotid arteries leads to the progression of Alzheimer's disease ${ }^{[64]}$. Osmotin protects against amyloid- $\beta$ deposition, tau phosphorylation, and apoptotic neurodegeneration through AdipoR1 followed by the AMPK/ sirtuin 1 /sterol regulatory element-binding protein 2 pathway in neuronal cells ${ }^{[25,65-67]}$. It also enhances neurite outgrowth and synaptic complexity via AdipoR1/nogo-66 receptor NgR1 signaling ${ }^{[68]}$. These findings indicate that osmotin is a potential candidate for the treatment of neurological disorders such as Alzheimer's disease. A preclinical trial study recently reported the usefulness of intravenous administration of osmotin-loaded magnetic nanoparticles in combination with electromagnetic guidance in the treatment of Alzheimer's disease ${ }^{[69]}$. These effects of osmotin are compatible with neuroprotective effects of adiponectin and AdipoRon in Alzheimer's disease $\mathrm{e}^{[70-73]}$.

\section{ANTI-TUMORIGENESIS EFFECT}

Cancer and atherosclerosis have been classified as non-communicable diseases by differing in target cells $^{[74]}$. However, both diseases have principally identical mechanisms such as cell proliferation induced by inflammation and reactive oxygen species ${ }^{[75]}$. Adiponectin induces anti-angiogenesis and anti-tumor activity through AdipoR1 via caspase-mediated EC apoptosis ${ }^{[13]}$. Actually, adiponectin and AdipoRon suppress the proliferation of a variety of human cancer cells ${ }^{[76-78]}$. Similarly, osmotin induces cell cycle arrest in the Go/G1 phase ${ }^{[33]}$ and suppresses cell proliferation ${ }^{[10]}$. These findings suggest the possibility that osmotin may suppress tumor growth. However, further studies are needed to clarify this hypothesis.

\section{THERAPEUTIC STRATEGY}

Osmotin is a natural plant protein that is ubiquitous in fruits and vegetables ${ }^{[13]}$. Osmotin is also known to be a phytochemical that is resistant to heat treatment (cooking) ${ }^{[13]}$. Furthermore, the osmotin protein is relatively stable and may maintain activity even through contact with the human digestive system ${ }^{[13]}$. Therefore, this phytochemical could be administered by ingestion of fruits and vegetables containing a great amount of osmotin or by oral administration of osmotin and/or its analogs that consist of the amino acid residue including an active center ${ }^{[10]}$. However, the best drug delivery system (DDS) for osmotin administration is considered to be enveloped in nanocapsules in order to avoid digestive degradation ${ }^{[10,69]}$. Moreover, continuing preclinical and clinical investigations with osmotin, in particular combined with a nanocapsule system as carrier vehicles into atherosclerotic lesions, are essential for future studies ${ }^{[10]}$. It is very important for DDS establishment to design the size of the nanocapsule so that it can go through a relatively wide gap between damaged vascular ECs, but not a normal gap between intact ECs in humans. The magnetic nanocapsule-based DDS with external electromagnetic guidance may be more useful to make osmotin cross endothelial gap junction ${ }^{[69]}$. Future studies are needed to clarify whether oral administration of osmotin-loaded nanocapsules can treat vascular lesions in patients with atherosclerosis. Similarly, osmotin-like proteins derived from many vegetables and fruits are also regarded as adiponectin peptidomimetics and gather attention as novel therapeutic drug candidates for atherosclerosis and related diseases $^{[79]}$.

\section{CONCLUSION}

These findings indicate that the novel phytochemical osmotin might be an effective therapeutic agent for atherosclerosis, inflammation, neurodegeneration, and their related diseases and that AdipoR1 might be a crucial therapeutic target for these diseases. Osmotin may open up a new therapeutic window in the treatment of atherosclerosis in cases with hypoadiponectinemia and/or resistance against adiponectin and AdipoRon $^{[10,30,80]}$. Osmotin also provides benefits to maintain vascular health and prevent vascular disease in healthy individuals. 


\section{DECLARATIONS}

\section{Acknowledgments}

The author thanks Ms. Yui Takahashi for her technical assistance.

\section{Authors' contributions}

The author contributed solely to the article.

\section{Availability of data and materials}

Not applicable.

\section{Financial support and sponsorship}

This work was supported in part by a Grant-in-Aid for Scientific Research (C) (17Ko8993 to T.W.) from the Japan Society for the Promotion of Science.

\section{Conflicts of interest}

The author declared that there are no conflicts of interest.

\section{Ethical approval and consent to participate}

Not applicable.

\section{Consent for publication}

Not applicable.

\section{Copyright}

(c) The Author(s) 2020.

\section{REFERENCES}

1. Herrington W, Lacey B, Sherliker P, Armitage J, Lewington S. Epidemiology of atherosclerosis and the potential to reduce the global burden of atherothrombotic disease. Circ Res 2016;118:535-46.

2. Tuttolomondo A, Di Raimondo D, Pecoraro R, Arnao V, Pinto A, et al. Atherosclerosis as an inflammatory disease. Curr Pharm Des 2012;18:4266-88.

3. Bentzon JF, Otsuka F, Virmani R, Falk E. Mechanisms of plaque formation and rupture. Circ Res 2014;114:1852-66

4. Jian X, Liu Y, Zhao Z, Zhao L, Wang D, et al. The role of traditional Chinese medicine in the treatment of atherosclerosis through the regulation of macrophage activity. Biomed Pharmacother 2019;118:109375.

5. Malekmohammad K, Sewell RDE, Rafieian-Kopaei M. Antioxidants and atherosclerosis: mechanistic aspects. Biomolecules 2019;9:301.

6. Singh S. Herbal approach for management of atherosclerosis: a review. Curr Atheroscler Rep 2019;21:12.

7. Wang C, Niimi M, Watanabe T, Wang Y, Liang J, et al. Treatment of atherosclerosis by traditional Chinese medicine: questions and quandaries. Atherosclerosis 2018;277:136-44.

8. Gholipour S, Sewell RDE, Lorigooini Z, Rafieian-Kopaei M. Medicinal plants and atherosclerosis: a review on molecular aspects. Curr Pharm Des 2018;24:3123-31.

9. Sedighi M, Bahmani M, Asgary S, Beyranvand F, Rafieian-Kopaei M. A review of plant-based compounds and medicinal plants effective on atherosclerosis. J Res Med Sci 2017;22:30.

10. Takahashi Y, Watanabe R, Sato Y, Ozawa N, Kojima M, et al. Novel phytopeptide osmotin mimics preventive effects of adiponectin on vascular inflammation and atherosclerosis. Metabolism 2018;83:128-38.

11. Liu J, Sui H, Zhao J, Wang Y. Osmotin protects H9c2 cells from stimulated ischemia-reperfusion injury through AdipoR1/PI3K/AKT signaling pathway. Front Physiol 2017;8:611.

12. Singh NK, Bracker CA, Hasegawa PM, Handa AK, Buckel S, et al. Characterization of osmotin: a thaumatin-like protein associated with osmotic adaptation in plant cells. Plant Physiol 1987;85:529-36

13. Anil Kumar S, Hima Kumari P, Shravan Kumar G, Mohanalatha C, Kavi Kishor PB. Osmotin: a plant sentinel and a possible agonist of mammalian adiponectin. Front Plant Sci 2015;6:163.

14. Viktorova J, Krasny L, Kamlar M, Novakova M, Mackova M, et al. Osmotin, a pathogenesis-related protein. Curr Protein Pept Sci 2012;13:672-81

15. Liu D, Narasimhan ML, Xu Y, Raghothama KG, Hasegawa PM, et al. Fine structure and function of the osmotin gene promoter. Plant 
Mol Biol 1995;29:1015-26.

16. Viktorova J, Rehorova K, Musilova L, Suman J, Lovecka P, et al. New findings in potential applications of tobacco osmotin. Protein Expr Purif 2017;129:84-93.

17. Yun DJ, Ibeas JI, Lee H, Coca MA, Narasimhan ML, et al. Osmotin, a plant antifungal protein, subverts signal transduction to enhance fungal cell susceptibility. Mol Cell 1998;1:807-17.

18. Narasimhan ML, Coca MA, Jin J, Yamauchi T, Ito Y, et al. Osmotin is a homolog of mammalian adiponectin and controls apoptosis in yeast through a homolog of mammalian adiponectin receptor. Mol Cell 2005;17:171-80.

19. Ohashi K, Ouchi N, Matsuzawa Y. Anti-inflammatory and anti-atherogenic properties of adiponectin. Biochimie 2012;94:2137-42.

20. Sargolzaei J, Chamani E, Kazemi T, Fallah S, Soori H. The role of adiponectin and adipolin as anti-inflammatory adipokines in the formation of macrophage foam cells and their association with cardiovascular diseases. Clin Biochem 2018;54:1-10.

21. Yamauchi T, Kadowaki T. Physiological and pathophysiological roles of adiponectin and adiponectin receptors in the integrated regulation of metabolic and cardiovascular diseases. Int J Obes (Lond) 2008;32:S13-8.

22. Miele M, Costantini S, Colonna G. Structural and functional similarities between osmotin from Nicotiana tabacum seeds and human adiponectin. PLoS One 2011;6:e16690.

23. Aouida M, Kim K, Shaikh AR, Pardo JM, Eppinger J, et al. A Saccharomyces cerevisiae assay system to investigate ligand/AdipoR1 interactions that lead to cellular signaling. PLoS One 2013;8:e65454.

24. Badshah H, Ali T, Kim MO. Osmotin attenuates LPS-induced neuroinflammation and memory impairments via the TLR4/NFKB signaling pathway. Sci Rep 2016;6:24493.

25. Naseer MI, Ullah I, Narasimhan ML, Lee HY, Bressan RA, et al. Neuroprotective effect of osmotin against ethanol-induced apoptotic neurodegeneration in the developing rat brain. Cell Death Dis 2014;5:e1150.

26. de Winther MPJ, Kanters E, Kraal G, Hofker MH. Nuclear factor kappaB signaling in atherogenesis. Arterioscler Thromb Vasc Biol 2005;25:904-14.

27. Muslin AJ. MAPK signaling in cardiovascular health and disease: molecular mechanisms and therapeutic targets. Clin Sci (Lond) 2008;115:203-18.

28. Motoshima H, Goldstein BJ, Igata M, Araki E. AMPK and cell proliferation-AMPK as a therapeutic target for atherosclerosis and cancer. J Physiol 2006;574:63-71.

29. Fang H, Judd R. Adiponectin regulation and function. Compr Physiol 2018;8:1031-63.

30. Esfahani M, Shabab N, Saidijam M. AdipoRon may be benefit for atherosclerosis prevention. Iran J Basic Med Sci 2017;20:107-9.

31. Trivedi VR, Chorawala MR, Shah GB. Antiatherosclerotic activity of osmotin, an adiponectin agonist in atherogenic diet induced hypertriglyceridemia and hypercholesterolemia in Wistar rats. Adv Res Pharmaceuticals Biol 2012;2:196-207.

32. Trivedi VR, Chorawala MR, Shah GB. Osmotin: a new adiponectin agonist, in type-II diabetes and obesity. Int J Pharm Sci Rev Res 2012;16:70-4.

33. Jo MG, Kim MW, Jo MH, Bin Abid N, Kim MO. Adiponectin homolog osmotin, a potential ant-obesity compound, suppresses abdominal fat accumulation in C57BL/6 mice on high-fat diet and in 3T3-L1 adipocytes. Int J Obes (Lond) 2019;43:2422-33.

34. Zhao W, Wu C, Li S, Chen X. Adiponectin protects palmitic acid induced endothelial inflammation and insulin resistance via regulating ROS/IKK pathways. Cytokine 2016;88:167-76.

35. Ouchi N, Kihara S, Arita Y, Maeda K, Kuriyama H, et al. Novel modulator for endothelial adhesion molecules: adipocyte-derived plasma protein adiponectin. Circulation 1999;100:2473-6.

36. Lu Y, Gao X, Wang R, Sun J, Guo B, et al. Adiponectin inhibits proliferation of vascular endothelial cells induced by Ox-LDL by promoting dephosphorylation of Caveolin-1 and depolymerization of eNOS and up-regulating release of NO. Int Immunopharmacol 2019;73:424-34.

37. Ohashi K, Parker JL, Ouchi N, Higuchi A, Vita JA, et al. Adiponectin promotes macrophage polarization towards an anti-inflammatory phenotype. J Biol Chem 2010;285:6153-60.

38. Wulster-Radcliffe MC, Ajuwon KM, Wang J, Christian JA, Spurlock ME. Adiponectin differentially regulates cytokines in porcine macrophages. Biochem Biophys Res Commun 2004;316:924-9.

39. Ouchi N, Kihara S, Arita Y, Nishida M, Matsuyama A, et al. Adipocyte-derived plasma protein, adiponectin, suppresses lipid accumulation and class A scavenger receptor expression in human monocyte-derived macrophages. Circulation 2001;103:1057-63.

40. Furukawa K, Hori M, Ouchi N, Kihara S, Funahashi T, et al. Adiponectin down-regulates acyl-coenzyme A:cholesterol acyltransferase-1 in cultured human monocyte-derived macrophages. Biochem Biophys Res Commun 2004;317:831-6.

41. Tsubakio-Yamamoto K, Matsuura F, Koseki M, Oku H, Sandoval JC, et al. Adiponectin prevents atherosclerosis by increasing cholesterol efflux from macrophages. Biochem Biophys Res Commun 2008;375:390-4.

42. Motobayashi Y, Izawa-Ishizawa Y, Ishizawa K, Orino S, Yamaguchi K, et al. Adiponectin inhibits insulin-like growth factor-1-induced cell migration by the suppression of extracellular signal-regulated kinase 1/2 activation, but not Akt in vascular smooth muscle cells. Hypertens Res 2009;32:188-93.

43. Chen X, Yuan Y, Wang Q, Xie F, Xia D, et al. Post-translational modulation of adiponectin affects lipid accumulation, proliferation and migration of vascular smooth muscle cells. Cell Physiol Biochem 2017;43:172-81.

44. Wang YT, Chen J, Li X, Umetani M, Chen Y, et al. Contribution of transcription factor EB to adipoRon-induced inhibition of arterial smooth muscle cell proliferation and migration. Am J Physiol Cell Physiol 2019;317:C1034-47.

45. Lu Y, Ma Y, Wang R, Sun J, Guo B, et al. Adiponectin inhibits vascular smooth muscle cell calcification induced by betaglycerophosphate through JAK2/STAT3 signaling pathway. J Biosci 2019;44:86. 
46. Kim HW, Belin de Chantemèle EJ, Weintraub NL. Perivascular adipocytes in vascular disease. Arterioscler Thromb Vasc Biol 2019;39:2220-7.

47. Ahmadieh S, Kim HW, Weintraub NL. Potential role of perivascular adipose tissue in modulating atherosclerosis. Clin Sci (Lond) 2020;134:3-13.

48. Pandey GK, Vadivel S, Ranghavan S, Mohan V, Balasubramanyam M, et al. High molecular weight adiponectin reduces glucolipotoxicity-induced inflammation and improves lipid metabolisim and insulin sensitivity via APPL1-AMPK-GLUT4 regulation in 3T3-L1 adipocytes. Atherosclerosis 2019;288:67-75.

49. Park M, Youn B, Zheng XL, Wu D, Xu A, et al. Globular adiponectin, acting via AdipoR1/APPL1. Protects H9c2 cells from hypoxia/ reoxygenation-induced apoptosis. PLoS One 2011;6:e19143.

50. Zhang Y, Zhao J, Li R, Lau WB, Yuan YX, et al. AdipoRon, the first orally active adiponectin receptor activator, attenuates postischemic myocardial apoptosis through both AMPK-mediated and AMPK-independent signaling. Am J Physiol Endocrinol Metab 2015;309:E275-82.

51. Okamoto Y, Kihara S, Ouchi N, Nishida M, Arita Y, et al. Adiponectin reduces atherosclerosis in apolipoprotein E-deficient mice. Circulation 2002;106:2767-70.

52. Yamauchi T, Kamon J, Waki H, Imai Y, Shimozawa N, et al. Globular adiponectin protected ob/ob mice from diabetes and ApoE-deficient mice from atherosclerosis. J Biol Chem 2003;278:2461-8.

53. Sun L, Yang X, Li Q, Zeng P, Liu Y, et al. Activation of adiponectin receptor regulates proprotein convertase subtilisin/kexin type 9 expression and inhibits lesions in ApoE-deficient mice. Arterioscler Thromb Vasc Biol 2017;37:1290-300.

54. Ahmad A, Ali T, Kim MW, Khan A, Jo MH, et al. Adiponectin homolog novel osmotin protects obesity/diabetes-induced NAFLD by upregulating AdipoRs/PPAR $\alpha$ signaling in ob/ob and db/db transgenic mouse models. Metabolism 2019;90:31-43.

55. Kashyap H, Gupta S, Bist R. Impact of active antihyperglycemic components as herbal therapy for preventive health care management of diabetes. Curr Mol Med 2019;19:12-9.

56. Ridker PM, Everett BM, Thuren T, MacFadyen JG, Chang WH, et al. Antiinflammatory therapy with Canakinumab for atherosclerotic disease. N Engl J Med 2017;377:1110-31.

57. Badshah H, Ali T, Kim MO. Osmotin attenuates LPS-induced neuroinflammation and memory impairments via the TLR4/NFKB signaling pathway. Sci Rep 2016;6:24493.

58. Arsenescu V, Narasimhan ML, Halide T, Bressan RA, Barisione C, et al. Adiponectin and plant-derived mammalian adiponectin homolog exert a protective effect in murine colitis. Dig Dis Sci 2011;56:2818-32.

59. Jian M, Kwan JS, Bunting M, Ng RC, Chan KH. Adiponectin suppresses amyloid- $\beta$ oligomer (A $\beta \mathrm{O})$-induced inflammatory response of microglia via AdipoR1-AMPK-NF-אB signaling pathway. J Neuroinflammation 2019;16:110.

60. Zhao Q, Liu Y, Tan L, Yan L, Zuo X. Adiponectin administration alleviates DSS-induced colonic inflammation in Caco-2 cells and mice. Inflamm Res 2018;67:663-70.

61. Wanleenuwat P, Iwanowski O, Kozubski W. Alzheimer's dementia: pathogenesis and impact of cardiovascular risk factors on cognitive decline. Postgrad Med 2019;131:415-22.

62. Lather R, Sapronova A, Kotelevtsev Y. Atherosclerosis and Alzheimer-diseases with a common cause? Inflammation, oxysterols, vasculature. BMC Geriatr 2014;14:36.

63. Fiolaki A, Tsamis KI, Milionis HJ, Kyritsis AP, Kosmidou M, et al. Atherosclerosis, biomarkers of atherosclerosis and Alzheimer's disease. Int J Neurosci 2014;124:1-11.

64. Xiang J. Carotid atherosclerosis promotes the progression of Alzheimer's disease: a three-year prospective study. Exp Ther Med 2017;14:1321-6.

65. Abid NB, Yoon G, Kim MO. Molecular cloning and expression of osmotin in a Baculovirus-insect system: purified osmotic mitigates amyloid-beta deposition in neural cells. Sci Rep 2017;7:8147.

66. Ali T, Yoon GH, Shah SA, Lee HY, Kim MO. Osmotin attenuates amyloid beta-induced memory impairment, tau phosphorylation and neurodegeneration in the mouse hippocampus. Sci Rep 2015;5:11708.

67. Shah SA, Yoon GH, Chung SS, Abid MN, Kim TH, et al. Novel osmotin inhibits SREBP2 via the AdipoR1/AMPK/SIRT1 pathway to improve Alzheimer's disease neuropathological deficits. Mol Psychiatry 2017;22:407-16.

68. Yoon G, Shah SA, Ali T, Kim MO. The adiponectin homolog osmotin enhances neurite outgrowth and synaptic complexity via AdipoR1/ NgR1 signaling in Alzheimer's disease. Mol Neurobiol 2018;55:6673-86.

69. Amin FU, Hoshiar AK, Do TD, Noh Y, Shah SA, et al. Osmotin-loaded magnetic nanoparticles with electromagnetic guidance for the treatment of Alzheimer's disease. Nanoscale 2017;9:10619-32.

70. Chan KH, Lam KS, Cheng OY, Kwan JS, Ho PW, et al. Adiponectin is protective against oxidative stress induced cytoxicity in amyloidbeta neurotoxicity. PLos One 2012;7:e52354.

71. Song J, Choi SM, Whitcomb DJ, Kim BC. Adiponectin controls the apoptosis and the expression of tight junction proteins in brain endothelial cells through AdipoR1 under beta amyloid toxicity. Cell Death Dis 2017;8:e3102.

72. Ng RC, Chan KH. Potential neuroprotective effects of adiponectin in Alzheimer's disease. Int J Mol Sci 2017;18:592.

73. Weisz F, Piccinin S, Mango D, Nqomba RT, Mercuri NB, et al. The role of adiponectin receptors in the regulation of synaptic transmission in the hippocampus. Synapse 2017;71:e21964.

74. Sima P, Vannucci L, Vetvicka V. Immunity in cancer and atherosclerosis. Ann Transl Med 2019;7:204.

75. Raposeiras Roubin S, Cordero A. The two-way relationship between cancer atherosclerosis. Rev Esp Cardiol (Engl Ed) 2019;72:487-94.

76. Jiang J, Fan Y, Zhang W, Shen Y, Liu T, et al. Adiponectin suppresses human pancreatic cancer growth through attenuating the $\beta$-catenin signaling pathway. Int J Biol Sci 2019;15:253-64. 
77. Nigro E, Stiuso P, Matera MG, Monaco ML, Caraglia M, et al. The anti-prolifeartive effects of adiponectin on human lung adenocarcinoma A549 cells and oxidative stress involvement. Pulm Pharmacol Ther 2019;55:25-30.

78. Ramzan AA, Bitler BG, Hicks D, Barner K, Qamar L, et al. Adiponectin receptor agonist AdipoRon induces apoptotic cell death and suppresses proliferation in human ovarian cancer cells. Mol Cell Biochem 2019;461:37-46.

79. Freitas CDT, Nishi BC, do Nascimento CTM, Silva MZR, Bezerra EHS, et al. Characterization of three osmotin-like proteins from plumeria rubra and prospection for adiponectin peptidomimetics. Protein Pept Lett 2020; Epub ahead of print. doi: 10.2174/09298665276 66200129154357

80. Iwabu M, Okada-Iwabu M, Yamauchi T, Kadowaki T. Adiponectin/AdipoR research and its implications for lifestyle-related diseases. Front Cardiovasc Med 2019;6:116. 